\title{
Efficient Simulation and Equilibrium Theory for Adsorption Processes with Implicit Adsorption Isotherms - Ideal Adsorbed Solution Theory
}

\author{
M. Fechtner ${ }^{\mathrm{a}}$, A. Kienle $\mathrm{a}^{\mathrm{a}, \mathrm{b}, *}$ \\ ${ }^{a}$ Otto-von-Guericke-Universität, Universitätsplatz 2, D-39106 Magdeburg, Germany \\ ${ }^{b}$ Max-Planck-Institut für Dynamik komplexer technischer Systeme, Sandtorstrasse 1, \\ D-39106 Magdeburg, Germany
}

\begin{abstract}
Recently an efficient method for the simulation of packed bed adsorbers with implicit adsorption isotherms was presented. It uses a method of lines approach and exploits standard software for the simultaneous solution of the resulting differential algebraic equations (DAEs). Application was demonstrated for stoichiometric ion exchange. In the present paper, the approach is extended to systems described by the adsorbed solution theory. For that purpose, the relation between the differential index of the DAE system and the spectral properties of the underlying adsorption equilibrium is established. In particular, it is shown that real and positive eigenvalues of the Jacobian of the underlying conservation equations will lead to a differential index of one. It is further shown that real and positive eigenvalues of the Jacobian related to the IAST can be guaranteed for binary mixtures with any type of pure component adsorption isotherm or for multicomponent mixtures with certain restricted types of pure component isotherms. The new method is illustrated for different explicit and implicit pure component adsorption isotherms belonging to this class. It is compared with alternative solution approaches using the modified FastIAS method by Do and Myers and semi-analytical solutions from equilibrium theory.
\end{abstract}

${ }^{*}$ Author to whom all correspondence should be addressed.

Email address: kienle@mpi-magdeburg.mpg.de (A. Kienle) 
Keywords: Adsorption, Ideal adsorbed solution theory, Simulation, Differential algebraic systems, Differential index

\section{Introduction}

Dynamic simulation of packed bed adsorbers plays an important role for the quantitative design of new single and multi bed adsorption processes. Therein, a detailed description of the adsorption equilibrium is of crucial importance. Particular challenging are implicit adsorption isotherms. For the efficient simulation of equilibrium models of packed bed adsorbers with implicit adsorption isotherms, we recently proposed a new approach [1]. It is based on a reformulation of the underlying partial differential equations, uses a method of lines (MOL) approach (see e.g. [2]), avoids explicit differentiation of the adsorption isotherm and applies standard numerics for the simultaneous solution of the

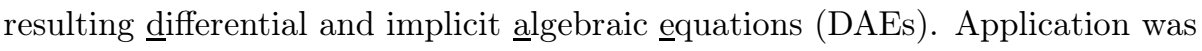
demonstrated for stoichiometric ion exchange. Focus was on single column operation. However, it is worth noting that the method is also very suitable for the simulation of multicolumn processes using available software packages for dynamic flow-sheet simulation like gProms [3], Diva [4] or Diana [5, 6] with a simultaneous equation oriented solution strategy.

In the present paper, the method is extended to another important type of adsorption equilibrium, i.e. the ideal adsorbed solution theory (IAST), which was developed by Myers and Prausnitz [7] to predict the adsorption of multicomponent mixtures from single component adsorption isotherms (SCIs), and which was successfully applied to gas (e.g. [8]) as well as liquid phase adsorption (e.g. 9]). The equations of the IAST are implicit and include integral expressions for the calculation of the spreading pressures. Only in special cases an analytical calculation of the equilibrium composition of the adsorbed phase is possible [10,11]. Therefore a number of numerical approaches were proposed including the general IAS [7], the FastIAS [12] and the modified FastIAS [12, 13], among others. For a recent review on available IAST solution approaches, we 
refer to [14] and [15]. All of these methods are based on iterative solution of the IAST equations, therefore require good initial guesses and almost entirely apply to analytically integrable explicit single component isotherms.

Therefore, an alternative approach was proposed recently by Landa et al. [14]. It is based on a differential reformulation of the equilibrium conditions and applies numerical integration. This method is very powerful and avoids iteration at the expense of increased computational effort. Further computational effort is required to calculate the derivatives of the equilibrium concentrations of the solid phase, which are needed for packed bed adsorber simulation using a method of lines approach [14].

To overcome the challenges and restrictions of previous approaches, the new methodology introduced in [1] is used and extended accordingly in the present paper. The outline of the paper is as follows: First, the model equations are briefly summarized and some structural properties that are essential for the new approach are proven. They are based on the spectral properties of the IAST equations and extend recent results by Landa et al. [14]. Afterwards application is demonstrated for two different benchmark problems with explicit and implicit SCIs. Results for explicit SCIs are compared to previous numerical solution approaches based on the modified FastIAS. In contrast to this, results for implicit SCIs are new and cannot be obtained with any of the previous approaches. In addition, numerical results for explicit and implicit SCIs are validated with semi-analytical solution approaches from equilibrium theory.

\section{Theoretical methods}

\subsection{Model equations}

The following is based on the equilibrium dispersive model, which is commonly used for describing the dynamics of fixed bed adsorbers [16, 17]. The model assumes isothermal operation, thermodynamic equilibrium between fluid phase with adsorbable components $\mathbf{c} \in \mathbb{R}^{N}$ and solid phase with adsorbed components $\mathbf{q} \in \mathbb{R}^{N}$, a constant mobile phase velocity $u$ and a constant void fraction 
$\epsilon$. These assumptions result in the following system of partial differential equations with time $t$ and spatial coordinate $z$

$$
F \frac{\partial \mathbf{q}(\mathbf{c})}{\partial t}+\frac{\partial \mathbf{c}}{\partial t}+u \frac{\partial \mathbf{c}}{\partial z}=D_{a} \frac{\partial^{2} \mathbf{c}}{\partial z^{2}} .
$$

Therein, $F=\frac{1-\epsilon}{\epsilon}$ is the volumetric phase ratio, and $D_{a}$ is an apparent dispersion coefficient lumping together all effects causing band broadening. For vanishing axial dispersion with $D_{a}=0$, the ideal equilibrium model is obtained from the above equation.

For a given adsorption isotherm $\mathbf{q}(\mathbf{c})$, given boundary and initial conditions, Eq. (1) can be solved for the unknown fluid phase concentrations c. In the present paper the adsorption isotherm is determined using the well known ideal adsorbed solution theory (IAST) [7, 8, 9]. The following equations provide a compact but complete representation of the IAST focusing on the essential variables and equations for this paper.

Single component isotherms (SCIs)

$$
q_{i}^{0}=q_{i}^{0}\left(c_{i}^{0}\right), \quad i=1, \ldots, N,
$$

equilibrium condition

$$
\int_{0}^{c_{i}^{0}} \frac{q_{i}^{0}(s)}{s} \mathrm{~d} s=\int_{0}^{c_{N}^{0}} \frac{q_{N}^{0}(s)}{s} \mathrm{~d} s, \quad i=1, \ldots, N-1,
$$

summation condition

$$
\sum_{j=1}^{N} \frac{c_{j}}{c_{j}^{0}}=1
$$

solid phase concentrations

$$
q_{i}=\left(\sum_{j=1}^{N} \frac{1}{q_{j}^{0}} \frac{c_{j}}{c_{j}^{0}}\right)^{-1} \frac{c_{i}}{c_{i}^{0}}, \quad i=1, \ldots, N .
$$

In theses equations, $\mathbf{c}^{0}$ and $\mathbf{q}^{0}$ denote the so-called hypothetical fluid and solid phase concentrations. $\mathbf{q}^{0}\left(\mathbf{c}^{0}\right)$ represents the given pure component isotherms and $\mathbf{c}^{0}$ is an additional implicit variable, which follows from Eqs. (3) and (4). For the details we refer to [7]. 


\subsection{Reformulation and solution strategy}

Besides the adsorption isotherms $\mathbf{q}(\mathbf{c})$, the solution of Eq. (1) also requires the Jacobian matrix of the derivatives of the adsorption isotherms $\partial \mathbf{q} / \partial \mathbf{c}$ according to the chain rule of differentiation. These derivatives however are difficult to obtain for complex adsorption isotherms. A method for the IAST based on some analytical formulas has been proposed in [14]. However, the method is tailored to the specific solution strategy in [14], which delivers a priori the hypothetical fluid phase concentrations $c_{i}^{0}$ as functions of the true fluid phase concentration $c_{i}$ and is not directly applicable to other solutions strategies. This motivates the following reformulation. A new set of variables $\mathbf{v} \in \mathbb{R}^{N}$ is introduced according to

$$
\mathbf{v}=F \mathbf{q}(\mathbf{c})+\mathbf{c}
$$

It represents the joint capacity of the fluid and the adsorbed phase in Eq. (11). In terms of the new variables the model (1) reads

$$
\frac{\partial \mathbf{v}}{\partial t}+u \frac{\partial \mathbf{c}}{\partial z}=D_{a} \frac{\partial^{2} \mathbf{c}}{\partial z^{2}}
$$

This equation is solved for $\mathbf{v}$. In addition, the following set of $2 N$ implicit algebraic equations for $\mathbf{c}^{0}$ and $\mathbf{c}$ is derived from Eqs. (2)-(5) and the definition of $\mathbf{v}$

$$
\begin{aligned}
0 & =f_{i}:=\int_{0}^{c_{i}^{0}} \frac{q_{i}^{0}(s)}{s} \mathrm{~d} s-\int_{0}^{c_{N}^{0}} \frac{q_{N}^{0}(s)}{s} \mathrm{~d} s, \quad i=1, \ldots, N-1, \\
0 & =f_{N}:=\sum_{j=1}^{N} \frac{c_{j}}{c_{j}^{0}}-1, \\
0 & =g_{i}:=F q_{i}(\mathbf{c})+c_{i}-v_{i} \\
& =F\left(\sum_{j=1}^{N} \frac{1}{q_{j}^{0}\left(c_{j}^{0}\right)} \frac{c_{j}}{c_{j}^{0}}\right)^{-1} \frac{c_{i}}{c_{i}^{0}}+c_{i}-v_{i} \quad i=1, \ldots, N .
\end{aligned}
$$


In the last equation, (5) was used to eliminate the $q_{i}$ variables.

For the numerical solution, the partial differential equation (7) is discretized using a method of lines approach [2]. For demonstration purposes the limiting case of vanishing axial dispersion is considered in the remainder, and a simple scheme with first order backward differences on an equidistant grid is used for the convection term. However, application of more efficient discretization methods using adaptive grids [18] and/or high resolution methods [19] is straight forward. The resulting system of ordinary differential equations (7) for $\mathbf{v}$ is solved simultaneously with algebraic equations (8)-(10) for $\mathbf{c}$ and $\mathbf{c}^{0}$ using standard DAE numerics like DASSL [20] or LIMEX [21] or ODE15s in Matlab[22] provided that the differential index is equal to one 23]. The numerical solution of higher index systems is much more challenging [24] and not required here as we will show subsequently. With the DAE approach explicit differentiation of the adsorption isotherm is avoided.

In the following, it is shown that the differential index of the PDAE system (7] 8, 10), or equivalently the DAE system resulting from its discretization, is always equal to one if the Jacobian $\partial \mathbf{q} / \partial \mathbf{c}$ has $N$ real, positive eigenvalues. For thermodynamic reasons this should always be the case as shown by Kvaalen et al. 25] using displacement theory. Explicit proofs for Langmuir isotherms were given in [25], for Bi-Langmuir isotherms in [26] and for the IAS theory for a large class of pure component isotherms in [14]. In the Appendix these results are extended in two directions. First, it is shown that this spectral property holds for any thermodynamically consistent adsorption isotherm for mixtures of two adsorbable components. Second, it is shown that it may fail for multicomponent mixtures that are not in the class defined by [14].

The PDAE system (7, 8, 10), or equivalently the DAE system resulting from its discretization, has differential index one if the matrix of the derivatives of the algebraic equations (8, 10 with respect to the algebraic variables $\mathbf{c}^{0}, \mathbf{c}$ is nonsingular. From differentiation of Eqs. (8, 10) and some elementary calculations we find 


$$
\begin{aligned}
\operatorname{det}(\mathbf{J}) & =\operatorname{det}\left(\begin{array}{cc}
\left(\frac{\partial \mathbf{f}}{\partial \mathbf{c}^{0}}\right)_{\mathbf{c}} & \left(\frac{\partial \mathbf{f}}{\partial \mathbf{c}}\right)_{\mathbf{c}^{0}} \\
\left(\frac{\partial \mathbf{g}}{\partial \mathbf{c}^{0}}\right)_{\mathbf{c}, \mathbf{v}} & \left(\frac{\partial \mathbf{g}}{\partial \mathbf{c}}\right)_{\mathbf{c}^{0}, \mathbf{v}}
\end{array}\right) \\
& =\operatorname{det}\left(\begin{array}{cc}
\left(\frac{\partial \mathbf{f}}{\partial \mathbf{c}^{0}}\right)_{\mathbf{c}} & \left.\left(\frac{\partial \mathbf{g}}{\partial \mathbf{c}}\right)_{\mathbf{c}^{0}, \mathbf{v}}-\left(\frac{\partial \mathbf{f}}{\partial \mathbf{c}}\right)_{\mathbf{c}^{0}}\right)_{\mathbf{c}, \mathbf{v}}\left(\frac{\partial \mathbf{f}}{\partial \mathbf{c}^{0}}\right)_{\mathbf{c}}^{-1}\left(\frac{\partial \mathbf{f}}{\partial \mathbf{c}}\right)_{\mathbf{c}^{0}}
\end{array}\right) \\
& =\operatorname{det}\left(\frac{\partial \mathbf{f}}{\partial \mathbf{c}^{0}}\right)_{\mathbf{c}} \operatorname{det}\left(\left(\frac{\partial \mathbf{g}}{\partial \mathbf{c}}\right)_{\mathbf{c}^{0}, \mathbf{v}}-\left(\frac{\partial \mathbf{g}}{\partial \mathbf{c}^{0}}\right)_{\mathbf{c}, \mathbf{v}}\left(\frac{\partial \mathbf{f}}{\partial \mathbf{c}^{0}}\right)_{\mathbf{c}}^{-1}\left(\frac{\partial \mathbf{f}}{\partial \mathbf{c}}\right)_{\mathbf{c}^{0}}\right) .
\end{aligned}
$$

The indices at the brackets indicate which variable is constant during differentiation. From this equation, we find that regularity of matrix $\mathbf{J}$ requires both determinants in the last row of this equation to be nonzero. The first determinant can be calculated explicitly using Gaussian elimination without row switching (see also [13])

$$
\begin{aligned}
\operatorname{det}\left(\left(\frac{\partial \mathbf{f}}{\partial \mathbf{c}^{0}}\right)_{\mathbf{c}}\right) & =\prod_{j=1}^{N-1} \frac{q_{j}^{0}}{c_{j}^{0}}\left(-\frac{c_{N}}{\left(c_{N}^{0}\right)^{2}}-\sum_{j=1}^{N-1} \frac{-\frac{c_{j}}{\left(c_{j}^{0}\right)^{2}}}{\frac{q_{j}^{0}}{c_{j}^{0}}}\left(-\frac{q_{N}^{0}}{c_{N}^{0}}\right)\right) \\
& =-q_{\text {tot }}^{-1} \prod_{j=1}^{N-1} \frac{q_{j}^{0}}{c_{j}^{0}} .
\end{aligned}
$$

It is nonzero for nonzero concentrations.

The matrix in the second determinant is equal to $\left(\frac{\partial \mathbf{g}}{\partial \mathbf{c}}\right)_{\mathbf{v}}$, which is obtained by differentiation of (10), when $\mathbf{c}^{0}$ is interpreted as a function of $\mathbf{c}$ according to Eqs. (819)

$$
\left(\frac{\partial \mathbf{g}}{\partial \mathbf{c}}\right)_{\mathbf{v}}=\left(\frac{\partial \mathbf{g}}{\partial \mathbf{c}}\right)_{\mathbf{c}^{0}, \mathbf{v}}+\left(\frac{\partial \mathbf{g}}{\partial \mathbf{c}^{0}}\right)_{\mathbf{c}, \mathbf{v}} \frac{\partial \mathbf{c}^{0}}{\partial \mathbf{c}}
$$

The derivative of $\mathbf{c}^{0}$ with respect to $\mathbf{c}$ is obtained by implicit differentiation of Eqs. (819) according to

$$
\frac{\partial \mathbf{c}^{0}}{\partial \mathbf{c}}=-\left(\frac{\partial \mathbf{f}}{\partial \mathbf{c}^{0}}\right)_{\mathbf{c}}^{-1}\left(\frac{\partial \mathbf{f}}{\partial \mathbf{c}}\right)_{\mathbf{c}^{0}} .
$$


Alternatively, $\left(\frac{\partial \mathbf{g}}{\partial \mathbf{c}}\right)_{\mathbf{v}}$ can be calculated by differentiation of the first line of Eq. (10)

$$
\left(\frac{\partial \mathbf{g}}{\partial \mathbf{c}}\right)_{\mathbf{v}}=F \frac{\partial \mathbf{q}}{\partial \mathbf{c}}+\mathbf{I}
$$

Hence, the second determinant in Eq. (11) yields

$$
\operatorname{det}\left(\frac{\partial \mathbf{g}}{\partial \mathbf{c}}\right)_{\mathbf{v}}=\prod_{i=1}^{N}\left(F \lambda_{i}+1\right),
$$

where the $\lambda_{i}$ 's are the eigenvalues of the matrix $\frac{\partial \mathbf{q}}{\partial \mathbf{c}}$. Consequently, the second determinant is also nonzero if these eigenvalues are positive, which completes the proof.

\section{Remarks:}

1. A crucial aspect of the DAE formulations are consistent initial values $\mathbf{v}_{0}(z), \mathbf{c}_{0}^{0}(z)$. They can be easily calculated by solving (8, 10, offline for given initial conditions $\mathbf{c}_{0}(z)$.

2. So far, explicit SCIs have been assumed according to equation (2). However, it is worth noting that also implicit SCIs according to

$$
0=h_{i}\left(c_{i}^{0}, q_{i}^{0}\right), \quad i=1, \ldots, N
$$

can be handled with the new method. For implicit SCI, equations (17) have to be solved for every $s \in\left[0, c_{i}^{0}\right]$, in order evaluate the integral expressions in (3). Since the hypothetical fluid phase concentrations $c_{i}^{0}$ depend on $z$ and $t$, this can be done in two different ways. Either the SCIs are reevaluated at every point in space and time according to the present values of $c_{i}^{0}$, or they are calculated a priori only once for estimated upper bounds on $c_{i}^{0}$, which is of course much more efficient. Using these function values, the integral expressions in (3) are then evaluated using some numerical quadrature.

The iterative calculation of the implicit SCIs requires good starting values, which are often not available. Therefore, the following approach based 
on integration according to Davidenko's method [27] with trivial initial conditions is used in this paper

$$
\frac{\mathrm{d} q_{i}^{0}}{\mathrm{~d} s_{i}}=-\left(\frac{\partial h_{i}}{\partial q_{i}^{0}}\right)^{-1} \frac{\partial h_{i}}{\partial s_{i}}, q_{i}^{0}(0)=0, i \in\{1, \ldots, N\} .
$$

3. In the limit of vanishing axial dispersion, an alternative reformulation of the partial differential equation (11) can be found. For this purpose Eqs. (2) and (5) are inserted into the partial differential equation (11) with $D_{a}=0$ leading to

$$
\begin{aligned}
& \frac{\partial \mathbf{c}}{\partial z}+u^{-1} \frac{\partial}{\partial t}\left(\mathbf{c}+F\left(\sum_{j=1}^{N} \frac{1}{q_{j}^{0}\left(c_{j}^{0}\right)} \frac{c_{j}}{c_{j}^{0}}\right) \mathbf{c} * \tilde{\mathbf{c}}^{0}\right)=\mathbf{0} \\
& \mathbf{c}(0, z)=\mathbf{c}_{0}(z), \mathbf{c}(t, 0)=\mathbf{c}_{i n j}(t) .
\end{aligned}
$$

In this equation the ' $*$ ' symbol denotes the element-wise multiplication of c and $\tilde{\mathbf{c}}^{0}=\left[\frac{1}{c_{1}^{0}}, \ldots, \frac{1}{c_{N}^{0}}\right]^{T}$. System (19) together with (8) and (9) forms a PDAE system that is equivalent to the previous system (77), and (8) 100). It can be solved efficiently using Rothe's Method [28] instead of a methods of lines (MOL) approach. In the Rothe method, first the temporal instead of the spatial coordinate is discretized leading to a system of ordinary differential equations in space, which are then integrated together with the algebraic equations using a DAE method. In this case, $\mathbf{c}$ is the dynamic variable and $\mathbf{c}^{0}$ is the only algebraic variable. This reformulation seems attractive because it requires only $N$ additional algebraic variables per grid point. In view of Eq. (12) the differential index of this alternative reformulation is also always equal to one. Hence, the Rothe-based DAE formulation is applicable to any SCIs, especially also to those resulting in non-hyperbolic systems. This allows to retain the advantages of a DAE formulation (numerical stability, usage of flow-sheet simulation) also for cases where the MOL-based DAE formulation is not applicable. 


\section{Applications}

In this section, the methods introduced in the previous section are evaluated and compared to established solution approaches for two different benchmark problems with explicit and implicit SCIs, respectively. For a simple and efficient validation with the equilibrium theory, binary examples are considered. However, based on the formulation and results in the previous chapter, application of the developed numerical solution approaches to multicomponent systems is straight forward.

The numerical results in this section were obtained using a standard desktop computer with intel ${ }^{\circledR} \operatorname{core}^{\mathrm{TM}} \mathrm{i} 7-47903.6 \mathrm{GHz}$ and 16 GB RAM. Matlab ${ }^{\circledR}$ [29] was used to perform the numerical simulations. In particular, ODE15s was used to solve the DAEs resulting from the discretization of the partial differential equations using first order backward differences, ODE45 to solve the ODEs resulting from Davidenko's method in Eq. (18) and the Matlab function 'trapz' for numerical evaluation of the integrals in Eq. (3). In addition to ODE15s the open source solver IDAS was tested as an alternative. This solver was incorporated into the Matlab ${ }^{\circledR}$ environment using SUNDIALS: Suite of nonlinear and differential/algebraic equation solvers [30].

\subsection{Example with explicit SCIs}

The first example was taken from [14]. It is concerned with the adsorption of phenyl-n-decane (abbreviation 'C10') and phenyl-n-undecane (abbreviation 'C11') in acetonitrile on graphitized carbon. Adsorption of individual components is described by explicit SCIs using a combined quadratic plus Langmuir model

$$
q_{i}^{0}=q_{i 1}^{s a t} \frac{c_{i}^{0}\left(b_{i 1}+2 b_{i 2} c_{i}^{0}\right)}{1+b_{i 1} c_{i}^{0}+b_{i 2}\left(c_{i}^{0}\right)^{2}}+q_{i 2}^{s a t} \frac{c_{i}^{0} b_{i 3}}{1+b_{i 3} c_{i}^{0}}, \quad i=1, \ldots N .
$$

Parameter values were taken from [14] and can be found in Tab. [1. The SCIs are shown in Fig. 1 Since this is a system with two adsorbable components, 
requirements for a differential index of 1 as discussed in the previous section and the Appendix are satisfied.

For a rigorous evaluation, the new MOL-based and Rothe-based DAE approaches are compared to two different implementations of the modified FastIAS by Do [13], which was originally developed by [12]. The modified FastIAS solves efficiently the equilibrium equations (819) using Newton method. The first implementation is a mixed DAE-FastIAS approach that uses the reformulation (7), (10) in combination with a method of lines approach and the modified FastIAS for the calculation of the hypothetical fluid phase concentrations $c_{i}^{0}$. Thus, solving simultaneously equations (77),(10) for $\mathbf{v}$, $\mathbf{c}$, while the equations (819) are solved separately for each spatial grid point at any given time for $\mathbf{c}^{0}$. Again, in light of Eq. (16) the differential index of this DAE approach is also equal to one. It is worth pointing out that in the new DAE approaches, all algebraic equations are solved simultaneously with the differential equations using DAE numerics, whereas in the modified FastIAS approaches Eqs. (10) are solved separately by iteration. Therefore the new MOLand Rothe-based approaches are expected to be more efficient and stable.

For the second implementation of the modified FastIAS method, total discretization of the model (11) is applied using simple backward differences for the spatial derivatives and forward differences for the time derivatives. Again the modified FastIAS method is used at any point in time and space. In all cases axial dispersion is neglected with $D_{a}=0$.

In the remainder, focus is on the following scenario. An empty column is injected with a pulse feed of $5.4\left[\frac{\mathrm{mmol}}{\mathrm{l}}\right]$ phenyl-n-decane and $5.0\left[\frac{\mathrm{mmol}}{\mathrm{l}}\right]$ phenyln-undecane starting at $0 \mathrm{~min}$ and ending at $10 \mathrm{~min}$. The resulting breakthrough curves at the end of the column obtained with the different approaches over a time interval of $[0,20]$ min are shown in Fig. 2, Results are in good agreement with Fig. 8.4 in [15]. Corresponding computation times are given in Tab. 2,

Numerical parameters of the different approaches were selected in such a way that the accuracy of all approaches is similar and therefore allows a fair comparison of computation times. For the MOL-based DAE approach backward 
differences with $N_{z}=100$ equidistant space grid points were used. It was found that a four times higher number of equidistant time grid points was required for the Rothe-based DAE approach to achieve similar accuracy. Although the Rothe-based PDAE formulation (19/89) has only $2 N$ variables compared to $3 N$ variables for the MOL-based PDAE formulation (78/8] reported in Tab. 2 is higher due to the increased number of grid points. Further differences may be attributed to different structural properties of the different formulations. It is concluded that the Rothe-based approach is less attractive compared to the MOL approach and therefore not further considered in this paper.

From Tab. 2 it is also confirmed that our MOL-based DAE approach is much faster than the mixed DAE-FastIAS approach. The reasons have been discussed above.

Despite minor differences in accuracy, the total discretization in combination with the modified FastIAS is almost as efficient as our MOL-based DAE approach. For the total discretization, 400 time steps and 1250 space steps were used. The high number of space steps was required to satisfy the CFL condition for numerical stability [19], which is a crucial issue for the type of total discretization applied here. Main advantage of the MOL-based DAE approach compared to the total discretization approach are seen in two facts: (i) In the DAE approach, more sophisticated methods for time integration including variable order, variable step size in combination with error control can be applied without extra effort by using corresponding standard software. This may lead to improved numerical accuracy and stability. In case of the used IDAS solver, an improved efficiency is clearly visible, see Tab. 2. (ii) As stated above, the MOL-based DAE approach is particular well suited for the simulation of multicolumn processes using standard software for equation oriented dynamic flow-sheet simulation. Such tools support flexible configuration of complex plants from elementary modeling and/or process units and therefore reduce the implementation effort.

For validation purposes, the numerical solution of the MOL-based method 
is finally compared to the predictions of equilibrium theory in Fig. 3 [31]. Fig. 3a represents the solution of the scenario in Fig. 2 in the hodograph space of the eigenvectors of the Jacobian $\partial \mathbf{q} / \partial \mathbf{c}$. Eigenvalues and eigenvectors have been calculated with the formulas in the Appendix. The lower diagram represents an improved simulation with an increased number of $N_{z}=1000$ grid points to reduce the numerical dispersion. Agreement between theoretical and numerical results in Fig. 3a is excellent. The plateau values in Fig. 3b also coincide visibly with the corners of the trajectory in Fig. $3 \mathrm{a}$.

\subsection{Example with implicit $S C I s$}

Finally, application of the MOL-based DAE formulation is demonstrated for a second benchmark problem with implicit SCIs. Since the modified FastIAS needs integrable and explicit SCIs, it cannot be applied to this second example.

The example system was taken from [32]. It is concerned with the adsorption of 2-phenylethanol (component '1') and 3-phenylpropanol (component '2') in methanol-water on octadecyl-silica, where an implicit multicomponent Fowler-Guggenheim isotherm is used. In this paper we use the IAST based on the implicit SCIs obtained as limiting cases from the multicomponent FowlerGuggenheim isotherm

$$
0=h_{i}\left(c_{i}^{0}, q_{i}^{0}\right)=b_{i} c_{i}^{0} \exp \left(-\chi_{i} \frac{q_{i}^{0}}{q_{i}^{\text {sat }}}\right)+\frac{q_{i}^{0}}{q_{i}^{0}-q_{i}^{\text {sat }}}, \quad i=1,2 .
$$

Therefore, only a qualitative comparison with [32] is possible. Using the Davidenko procedure [27], equations (21) are replaced by the equivalent initial value problem

$$
\begin{aligned}
\frac{\mathrm{d} q_{i}^{0}}{\mathrm{~d} s} & =\left(\chi_{i} b_{i} \frac{s}{q_{i}^{s a t}} \exp \left(-\chi_{i} \frac{q_{i}^{0}}{q_{i}^{s a t}}\right)+\frac{q_{i}^{\text {sat }}}{\left(q_{i}^{0}-q_{i}^{\text {sat }}\right)^{2}}\right)^{-1} b_{i} \exp \left(-\chi_{i} \frac{q_{i}^{0}}{q_{i}^{s a t}}\right), \\
q_{i}(0) & =0, \quad i=1,2,
\end{aligned}
$$

to calculate the SCIs. The parameters for this example can be found in Tab. 3 and are taken from [32]. Results are shown in Fig. 4. Since the calculation 
of the SCIs can be separated from the simulation, the same arguments for the differential index can be applied as in the previous section and the Appendix. Again, the differential index is equal to one for the present system with two adsorbable components and the MOL-based DAE approach can be applied. For demonstration purposes axial dispersion is again neglected corresponding to $D_{a}=0$.

A similar set-up like the previous example is considered, where an empty column is injected with a feed pulse of $6.0 \frac{\mathrm{mg}}{\mathrm{ml}} 2$-phenylethanol and $6.0 \frac{\mathrm{mg}}{\mathrm{ml}} 3$ phenylpropanol starting at $t=0.0 \mathrm{~s}$ and ending at $t=10.0 \mathrm{~s}$. Corresponding breakthrough curves are shown in Fig. 5 for the time interval $[0,300] s$. Due to the different multicomponent isotherms, the results in Fig. 5 are only in qualitative agreement with Fig. 4 in [32]. Main difference is a shift of profiles by $25 \mathrm{~s}$. Nevertheless, the example in Fig. 5 demonstrates the applicability of the new MOL-based DAE approach also for non integrable and implicit single component adsorption isotherms.

In a second step, the numerical solution is also validated with the semianalytical solution obtained from equilibrium theory. For this purpose a chromatographic cycle consisting of the loading of an empty bed followed by the regeneration is considered. The solution trajectory in the hodograph space obtained from equilibrium theory is shown in Fig. 6 a. Corresponding concentration profiles calculated numerically with the MOL-based DAE approach using an increased number of $N_{z}=1000$ grid points is shown in Fig. 6b. Additionally, the injection interval is increased to $[0,100] s$ for an improved visibility of the plateau values. Again, agreement between theoretical and numerical results in Fig. 6a is excellent. In the concentration range considered in this figure, the system is almost linear decoupled. This is reflected by the almost orthogonal patch grid of the eigenvectors in Fig. $6 \mathrm{~b}$ and by the decoupled transitions as well as the 'symmetry' between adsorption and desorption fronts in Fig. 6b. 


\section{Conclusion}

Two different DAE-based methods were developed for the numerical solution of equilibrium models of fixed bed adsorbers for an arbritrary number of involved components, namely an MOL- and a Rothe-based approach. It was shown that the first is clearly superior to the second. Further, the efficiency of the MOLbased approach was verified by comparison with different implementations of the popular modified FastIAS method. Restrictions of (modified) FastIAS concerning the single component isotherms could be relaxed. It was shown that the DAE methods can be applied to a large class of SCIs including non integrable and even implicit equations. Applicability of the DAE approaches was justified by proving a differential index of one for all types of isotherms leading to a Jacobian of the derivatives of the adsorption isotherms with only real and positive eigenvalues. This applies to all binary and a large class of multicomponent mixtures. However, for multicomponent mixtures also cases with complex eigenvalues can be found, which are not suitable from the thermodynamic as well as the numerical point of view and need revision.

Validation was done for elementary single column operation and for mixtures with relatively simple thermodynamic behavior. Future work will focus on more complex processes and/or more complex thermodynamic patterns of behavior.

\section{Acknowledgments.}

The financial support of the International Max Planck Research School for Advanced Methods in Process and Systems Engineering - IMPRS ProEng is greatly acknowledged. 


\section{Notation}

\begin{tabular}{|lll|}
\hline$b_{i j}$ & specific linear isotherm parameter & {$[\cdot]$} \\
$c_{i}$ & fluid phase concentration of component i & {$\left[\frac{\mathrm{mol}}{\mathrm{m}^{3}}\right]$} \\
$c_{i}^{0}$ & hypothetical fluid phase concentration of component i & {$\left[\frac{\mathrm{mol}}{\mathrm{m}^{3}}\right]$} \\
$D_{a}$ & apparent dispersion coefficient & {$\left[\frac{\mathrm{m}^{2}}{\mathrm{~s}}\right]$} \\
$F$ & volumetric phase ratio & {$[-]$} \\
$f_{i}$ & i-th algebraic function of the IAST & {$[-]$} \\
$g_{i}$ & i-th artificially algebraic function & {$[-]$} \\
$h_{i}$ & i-th implicit SCI & {$[-]$} \\
$\mathbf{I}$ & identity matrix of size $N \times N$ & {$[-]$} \\
$J_{i j}$ & i-th row, j-th column of the adsorption related Jacobian & {$[-]$} \\
$L_{c}$ & column length & {$[\mathrm{m}]$} \\
$N$ & number of components & {$[-]$} \\
$N_{z}$ & number of grid points & {$[-]$} \\
$q_{i}$ & solid phase concentration of component i & {$\left[\frac{\mathrm{mol}}{\mathrm{m}^{3}}\right]$} \\
$q_{t o t}$ & total solid phase concentration & {$\left[\frac{\mathrm{mol}}{\mathrm{m}^{3}}\right]$} \\
$q_{i}^{0}$ & hypothetical solid phase concentration of component i & {$\left[\frac{\mathrm{mol}}{\mathrm{m}^{3}}\right]$} \\
$q_{i}^{\text {sat }}$ & specific saturation isotherm parameter & {$[\cdot]$} \\
$r_{i}$ & specific exponential isotherm parameter & {$[-]$} \\
$s$ & integration/differentiation variable & {$\left[\frac{\mathrm{mol}}{\mathrm{m}^{3}}\right]$} \\
$t$ & time coordinate & {$[\mathrm{s}]$} \\
$u$ & mobile phase velocity & {$\left[\frac{\mathrm{m}}{\mathrm{s}}\right]$} \\
$v_{i}$ & joint capacity of fluid and solid phase of component i & {$\left[\frac{\mathrm{mol}}{\mathrm{m}^{3}}\right]$} \\
$x_{i}$ & molar fraction of component i & {$[-]$} \\
$z$ & space coordinate & {$[\mathrm{m}]$} \\
\hline$\epsilon$ & void fraction & {$[-]$} \\
$\lambda_{i}$ & i-th eigenvalue of the Jacobian \\
$\chi_{i}$ & specific mixed linear-exponential isotherm parameter & {$[-]$} \\
$\omega$ & denominator function of specific SCIs & {$[-]$} \\
\hline & & {$[\cdot]$} \\
\hline
\end{tabular}




\section{References}

[1] M. Fechtner, A. Kienle, Efficient simulation and equilibrium theory for adsorption processes with implicit adsorption isotherms - mass action equilibria, Chemical Engineering Science 171 (2017) 471-480. doi:10.1016/j.ces.2017.06.004.

[2] W. E. Schiesser, The Numerical Method of Lines Integration of Partial Differential Equations, Academic Press, San Diego, 1991.

[3] Process Systems Enterprise, gPROMS, www.psenterprise.com/gproms (1997-2017).

[4] M. Mangold, A. Kienle, K. D. Mohl, E. D. Gilles, Nonlinear computation using DIVA - Methods and applications, Chemical Engineering Science 55 (2000) 441-454.

[5] M. Krasnyk, K. Bondareva, O. Milokhov, K. Teplinskiy, M. Ginkel, A. Kienle, The promot/diana simulation environment, Computer Aided Chemical Engineering 21 (2006) 445-450.

[6] M. Krasnyk, M. Ginkel, M. Mangold, A. Kienle, Numerical analysis of higher order singularities in chemical process models, Computers \& Chemical Engineering 31 (2007) 1100-1110.

[7] A. Myers, J. Prausnitz, Thermodynamics of mixed-gas adsorption, AIChE J. 11 (1965) 121-127. doi:10.1002/aic.690110125.

[8] E. N. Rudisill, M. D. LeVan, Standard states for the adsorbed-solution theory, Chem. Eng. Sci. 47(5) (1992) 1239-1245.

[9] C. J. Radke, J. M. Prausnitz, Thermodynamics of multi-solute adsorption from dilute liquid solutions, AIChE J. 18(4) (1972) 761-768. doi:10.1002/aic.690180417. 
[10] M. Ilic, D. Flockerzi, A. Seidel-Morgenstern, A thermodynamically consistent explicit competitive adsorption isotherm model based on second order single component behavior, Journal of Chromatography A 1217 (2010) 2132-2137.

[11] A. Tarafder, M. Mazzotti, A method for deriving explicit binary isotherms obeying the ideal adsorbed solution theory, Chemical Engineering Technology 35 (2012) 102-108.

[12] J. O'Brien, A. Myers, A comprehensive technique for equilibrium calculations in adsorbed mixtures: The generalized fastias method, Ind. Eng. Chem. Res. 27 (1988) 2085-2092. doi:10.1021/ie00083a021.

[13] D. D. Do, Adsorption Analysis: Equilibria and Kinetics, Vol. 2 Series on Chemical Engineering, Imperial College Press, London, 1998.

[14] H. Landa, D. Flockerzi, A. Seidel-Morgenstern, A method for efficiently solving the iast equations with an application to adsorber dynamics, AIChE J. 59 (2013) 1263-1277. doi:10.1002/aic.13894.

[15] H. Landa, Development of an efficient method for simulating fixed-bed adsorption dynamics using ideal adsorbed solution theory, Ph.D. thesis, Otto-von-Guericke-University Magdeburg (2016).

[16] G. Guiochon, A. Felinger, D. G. Shirazi, A. M. Katti, Fundamentals of Preparative and Nonlinear Chromatography, Vol. 2, Elsevier Academic Press, San Diego, 2006.

[17] G. Guiochon, B. Lin, Modeling for Preparative Chromatography, Vol. 1, Elsevier Academic Pressl, San Diego, 2003.

[18] R. Köhler, K. D. Mohl, H. Schramm, M. Zeitz, A. Kienle, M. Mangold, E. Stein, E. D. Gilles, Methods of lines within the simulation enviroment DIVA for chemical processes, in: A. VandeWouwer, P. Saucez, W. Schiesser (Eds.), Adaptive method of lines, CRC Press, 2001, pp. 371-406. 
[19] R. J. LeVeque, Numerical Methods for Conservation Laws, Birkhäuser Verlag, Basel, 1992.

[20] K. E. Brenan, S. L. Campbell, L. R. Petzold, Numerical Solution of Initial Value Problems in Differential-Algebraic Equations, North Holland \& Elsevier Science Publishing Company, 1989.

[21] P. Deufhard, E. Hairer, J. Zugck, One-step and extrapolation methods for differential-algebraic systems, Numerische Mathematik 51 (1987) 501-516.

[22] MATLAB, version 8.4.0 (R2014b), The MathWorks Inc., Natick, Massachusetts, 2014.

[23] J. Unger, A. Kröner, W. Marquardt, Structural analysis of differentialalgebraic equation systems: Theory and applications, Computers \& Chemical Engineering 19 (1995) 867-882.

[24] P. Kunkel, V. Mehrmann, Differential-algebraic Equations: Analysis and Numerical Solution, European Mathematical Society, Switzerland, Zürich, 2006.

[25] E. Kvaalen, L. Neel, D. Tondeur, Directions of quasi-static mass and energy transfer between phases in multicomponent open systems, Chemical Engineering Science 40 (1985) 1191-1204.

[26] D. Flockerzi, M. Kaspereit, A. Kienle, Spectral properties of bilangmuir isotherms, Chemical Engineering Science 104 (2013) 957-959. doi:10.1016/j.ces.2013.09.045

[27] R. Deydel, Practical Bifurcation and Stability Analysis, Springer-Verlag, New York, 1994.

[28] P. Knabner, L. Angermann, Numerical Methods for Elliptic and Parabolic Partial Differential Equations, Springer-Verlag, New York, 2003.

[29] I. M. The MathWorks, Version 9.0.0.341360 (R2016a), Birkhäuser Verlag, Natick, Massachusetts, USA, 2016. 
[30] A. Hindmarsh, P. Brown, K. Grant, S. Lee, R. Serban, D. Shumaker, C. Woodward, SUNDIALS: Suite of nonlinear and differential/algebraic equation solvers, ACM Transactions on Mathematical Software (TOMS) 31 (3) (2005) 363-396.

[31] H.-K. Rhee, R. Aris, N. R. Amundson, First-Order Partial Differential Equations: Volume II - Theory and Application of Hyperbolic Systems of Quasilinear Equations, Prentice Hall, New Jersey, 1989.

[32] K. Kaczmarski, D. Antos, Calculation of chromatographic band profiles with an implicit isotherm, J. Chromatogr. A 862(1) (1999) 1-16. doi:10.1016/S0021-9673(99)00901-2

[33] M. Mazzotti, A. Rajendran, Equilibrium theory-based analysis of nonlinear waves in separation processes, Annual Review of Chemical and Biomolecular Engineering 4 (2013) 119-141.

[34] K. V. Kumar, M. M. de Castro, M. Martinez-Escandell, M. MolinaSabio, F. Rodriguez-Reinoso, A site energy distribution function from toth isotherm for adsorption of gases on heterogeneous surfaces, Phys. Chem. Chem. Phys. 13 (2011) 5753-5759. doi:10.1039/C0CP00902D. 


\section{Appendix. Spectral properties of the IAST approach}

The results for the differential index in Section 2 depend on the eigenvalues $\lambda_{i}$ of the Jacobian matrix $\partial \mathbf{q} / \partial \mathbf{c}$. It has been shown in [14] that these eigenvalues are always real and positive for IAST isotherms provided the single component isotherms (SCI) $q_{i}^{0}\left(c_{i}^{0}\right)$ are strictly monotonically increasing and have the following structure

$$
q_{i}^{0}\left(c_{i}^{0}\right)=\frac{c_{i}^{0} \omega^{\prime}\left(c_{i}^{0}\right)}{\omega\left(c_{i}^{0}\right)} .
$$

This structural requirement is met by many but not all SCIs. A counter example is the Tóth isotherm, to be considered at the end of this appendix.

The monotonicity requirement

$$
\frac{\mathrm{d} q_{i}^{0}\left(c_{i}^{0}\right)}{\mathrm{d} c_{i}^{0}}>0, c_{i}^{0} \neq 0 \forall i \in\{1, \ldots, N\},
$$

is a direct consequence of phase stability. Any non monotonic SCI will give rise to two different fluid concentrations in equilibrium with the same adsorbed phase concentration indicating a phase split of the fluid phase.

It is worth noting that the above eigenvalue result implies hyperbolicity of the ideal equilibrium model without axial dispersion if the corresponding eigenvectors are linearly independent. Hyperbolicity is the basic requirement for the so called equilibrium theory [33], which allows a semi-analytical solution for piecewise constant initial and boundary conditions using the method of characteristics.

In this appendix the results from [14] will be extended in two directions. First, the result will be generalized to mixtures with two adsorbable components with any strictly monotonically increasing SCIs, no matter whether explicit or implicit. Therefore the structural requirement (A.1) is relaxed. Afterwards, it is shown that such general statement is not possible in the multicomponent case with more than two adsorbable components.

First focus is on binary mixtures. The Jacobian of the IAST approach takes 
with $\mathbf{c}$ being the only independent variable, i.e. $\mathbf{q}=\mathbf{q}\left(\mathbf{c}, \mathbf{c}^{0}(\mathbf{c}), \mathbf{q}^{0}\left(\mathbf{c}^{0}(\mathbf{c})\right)\right)$, the generalized form

$$
\begin{aligned}
\frac{\partial \mathbf{q}}{\partial \mathbf{c}} & =\left(\frac{\partial \mathbf{q}}{\partial \mathbf{c}}\right)_{\mathbf{c}^{0}}+\left(\frac{\partial \mathbf{q}}{\partial \mathbf{c}^{0}}\right)_{\mathbf{c}} \frac{\partial \mathbf{c}^{0}}{\partial \mathbf{c}}, \\
\left(\frac{\partial \mathbf{q}}{\partial \mathbf{c}^{0}}\right)_{\mathbf{c}} & =\left(\frac{\partial \mathbf{q}}{\partial \mathbf{c}^{0}}\right)_{\mathbf{c}, \mathbf{q}^{0}}+\left(\frac{\partial \mathbf{q}}{\partial \mathbf{q}^{0}}\right)_{\mathbf{c}, \mathbf{c}^{0}} \frac{\partial \mathbf{q}^{0}}{\partial \mathbf{c}^{0}}, \\
\frac{\partial \mathbf{c}^{0}}{\partial \mathbf{c}} & =-\left(\frac{\partial \mathbf{f}}{\partial \mathbf{c}^{0}}\right)_{\mathbf{c}}^{-1}\left(\frac{\partial \mathbf{f}}{\partial \mathbf{c}}\right)_{\mathbf{c}^{0}} .
\end{aligned}
$$

Note, in (A.3) we require the values of $q_{i}^{0}$ to be explicitly available for each $c_{i}^{0}$, but no further information on the functional structure of the $q_{i}^{0}$ is required, i.e. it does not matter if $q_{i}^{0}=q_{i}^{0}\left(c_{i}^{0}\right)$ or $0=h\left(q_{i}^{0}, c_{i}^{0}\right)$ is solved intermediately. A unique solution of the latter is guaranteed due to the strict monotonicity of the SCIs.

For $N=2$, the analytical formula of the Jacobian reads accordingly

$$
\begin{aligned}
& \mathbf{J}=\frac{\partial \mathbf{q}}{\partial \mathbf{c}}=q_{t o t}^{2}\left[\begin{array}{cc}
\frac{x_{2}}{c_{1}^{0} q_{2}^{0}}+M_{1} & -\frac{x_{1}}{c_{2}^{0} q_{2}^{0}}+\frac{c_{1}^{0}}{c_{2}^{0}} M_{1} \\
-\frac{x_{2}}{c_{1}^{0} q_{1}^{0}}+\frac{c_{2}^{0}}{c_{1}^{0}} M_{2} & \frac{x_{1}}{c_{2}^{0} q_{1}^{0}}+M_{2}
\end{array}\right], \\
& M_{1}=x_{1} \frac{q_{t o t}}{c_{1}^{0} q_{1}^{0}}\left(\frac{x_{1}}{q_{1}^{0}} \frac{c_{1}^{0}}{q_{1}^{0}} \frac{\mathrm{d} q_{1}^{0}}{\mathrm{~d} c_{1}^{0}}+\frac{q_{1}^{0}}{q_{2}^{0}} \frac{x_{2}}{q_{2}^{0}} \frac{c_{2}^{0}}{q_{2}^{0}} \frac{\mathrm{d} q_{2}^{0}}{\mathrm{~d} c_{2}^{0}}+\frac{q_{1}^{0}}{q_{2}^{0}} \frac{x_{2}}{q_{2}^{0}}-\frac{x_{2}}{q_{2}^{0}}\right), \\
& M_{2}=x_{2} \frac{q_{t o t}}{c_{2}^{0} q_{2}^{0}}\left(\frac{x_{2}}{q_{2}^{0}} \frac{c_{2}^{0}}{q_{2}^{0}} \frac{\mathrm{d} q_{2}^{0}}{\mathrm{~d} c_{2}^{0}}+\frac{q_{2}^{0}}{q_{1}^{0}} \frac{x_{1}}{q_{1}^{0}} \frac{c_{1}^{0}}{q_{1}^{0}} \frac{\mathrm{d} q_{1}^{0}}{\mathrm{~d} c_{1}^{0}}+\frac{q_{2}^{0}}{q_{1}^{0}} \frac{x_{1}}{q_{1}^{0}}-\frac{x_{1}}{q_{1}^{0}}\right),
\end{aligned}
$$

with molar fractions $x_{i}=c_{i} / c_{i}^{0}$. The eigenvalues of Jacobian in (A.4) can be derived from the characteristic equation $\operatorname{det}(\mathbf{J}-\lambda \mathbf{I})$ resulting in the following quadratic equation

$$
\begin{aligned}
& a \lambda^{2}-b \lambda+c=0, \\
& a=q_{\text {tot }}^{-4}, \\
& b=q_{\text {tot }}^{-2}\left(\frac{1}{c_{1}^{0}} \frac{1}{q_{2}^{0}} x_{2}+\frac{1}{c_{2}^{0}} \frac{1}{q_{1}^{0}} x_{1}+M_{1}+M_{2}\right), \\
& c=\frac{1}{c_{2}^{0}} \frac{1}{q_{1}^{0}} M_{1}+\frac{1}{c_{1}^{0}} \frac{1}{q_{2}^{0}} M_{2},
\end{aligned}
$$


where the two possible eigenvalues can be readily derived

$$
\lambda_{i}=\frac{b \pm \sqrt{b^{2}-4 a c}}{2 a} .
$$

In order to have a suitable spectral property that guarantees a differential of one for any pair monotonic increasing SCIs, both eigenvalues have to be real and positive. In A.5, the inequality $a>0$ is obviously satisfied since all concentrations are positive. Based on (A.6), the inequalities

$$
\begin{aligned}
& b>0, \\
& c>0, \\
& b^{2}-4 a c \geq 0,
\end{aligned}
$$

have to be additionally verified to realize the spectral property above.

Using relation (A.2), one can derive for both coefficients $a, b$ lower limits $a_{l}, b_{l}$ from their definition in (A.5) by applying $\frac{\mathrm{d} q_{i}^{0}}{\mathrm{~d} c_{i}^{0}}=0$ to $M_{1}$ and $M_{2}$ resulting in

$$
\begin{aligned}
& b_{l}=q_{\text {tot }}^{-1}\left(\frac{1}{c_{1}^{0} q_{2}^{0}} \frac{x_{2}}{q_{2}^{0}}+\frac{1}{c_{2}^{0} q_{1}^{0}} \frac{x_{1}}{q_{1}^{0}}\right)>0, \\
& b>b_{l}>0 \\
& c_{l}=\frac{q_{\text {tot }}}{c_{1}^{0} c_{2}^{0}}\left(\frac{x_{1} x_{2}}{q_{1}^{0} q_{2}^{0}}-\frac{x_{1} x_{2}}{\left(q_{1}^{0}\right)^{2} q_{2}^{0}}+\frac{x_{1} x_{2}}{\left(q_{1}^{0}\right)^{2} q_{2}^{0}}-\frac{x_{1} x_{2}}{q_{1}^{0} q_{2}^{0}}\right)=0, \\
& c>c_{l}=0 .
\end{aligned}
$$

The term $b^{2}-4 a c$ can be reformulated such that it readily satisfies the last inequality in (A.7) 


$$
\begin{aligned}
& b^{2}-4 a c=q_{t o t}^{-2}\left((\gamma-\alpha-\beta)^{2}+4(\mu-\nu)^{2} \xi\right) \geq 0, \\
& \alpha=\frac{1}{c_{1}^{0}} \frac{x_{1}}{q_{1}^{0}}\left(\frac{x_{1}}{q_{1}^{0}} \frac{c_{1}^{0}}{q_{1}^{0}} \frac{\mathrm{d} q_{1}^{0}}{\mathrm{~d} c_{1}^{0}}+\frac{q_{1}^{0}}{q_{2}^{0}} \frac{x_{2}}{q_{2}^{0}} \frac{c_{2}^{0}}{q_{2}^{0}} \frac{\mathrm{d} q_{2}^{0}}{\mathrm{~d} c_{2}^{0}}\right)>0, \\
& \beta=\frac{1}{c_{2}^{0}} \frac{x_{2}}{q_{2}^{0}}\left(\frac{x_{2}}{q_{2}^{0}} \frac{c_{2}^{0}}{q_{2}^{0}} \frac{\mathrm{d} q_{2}^{0}}{\mathrm{~d} c_{2}^{0}}+\frac{q_{2}^{0}}{q_{1}^{0}} \frac{x_{1}}{q_{1}^{0}} \frac{c_{1}^{0}}{q_{1}^{0}} \frac{\mathrm{d} q_{1}^{0}}{\mathrm{~d} c_{1}^{0}}\right)>0, \\
& \gamma=\frac{1}{c_{1}^{0} q_{2}^{0}} \frac{x_{2}}{q_{2}^{0}}+\frac{1}{c_{2}^{0} q_{1}^{0}} \frac{x_{1}}{q_{1}^{0}}>0, \\
& \mu=\frac{1}{c_{2}^{0} q_{1}^{0}}>0, \\
& \nu=\frac{1}{c_{1}^{0} q_{2}^{0}}>0, \\
& \xi=\frac{x_{1}}{q_{1}^{0}} \frac{x_{2}}{q_{2}^{0}}\left(x_{1} \frac{c_{1}^{0}}{q_{1}^{0}} \frac{q_{2}^{0}}{q_{1}^{0}} \frac{\mathrm{d} q_{1}^{0}}{\mathrm{~d} c_{1}^{0}}+x_{2} \frac{c_{2}^{0}}{q_{2}^{0}} \frac{q_{1}^{0}}{q_{2}^{0}} \frac{\mathrm{d} q_{2}^{0}}{\mathrm{~d} c_{2}^{0}}\right)>0,
\end{aligned}
$$

thus concluding the proof of $\lambda_{i} \in \mathbb{R}_{+}$with $i \in\{1,2\}$ for binary mixtures. If additionally the following is satisfied

$$
\int_{0}^{c_{1}^{0}} \frac{q_{1}^{0}(s)}{s} \mathrm{~d} s=\int_{0}^{c_{2}^{0}} \frac{q_{2}^{0}(s)}{s} \mathrm{~d} s \quad \rightarrow \quad \frac{q_{1}^{0}}{c_{1}^{0}} \neq \frac{q_{2}^{0}}{c_{2}^{0}}
$$

which is a negligible restriction for the choice of the SCIs, the last relations in (A.7) becomes a strict inequality since $(\mu-\nu)^{2}>0$ holds. Hence, both eigenvalues are also distinct and the Jacobian is strictly hyperbolic for nonvanishing concentrations.

The previous result of real and positive eigenvalues using only the assumption (A.2) cannot be extended to systems with more than two components, which is similar to the spectral results in [26]. For this purpose, a simple three component counter example using the Tóth isotherm

$$
q_{i}^{0}=q_{i}^{s a t} b_{i} c_{i}^{0}\left(1+\left(b_{i} c_{i}^{0}\right)^{r_{i}}\right)^{-\frac{1}{r_{i}}}, i \in\{1,2,3\}
$$

which satisfies (A.2) for all physically relevant concentrations, see also Fig. 7b, is considered. Parameter values in Tab. 4 are taken from [34]. A reasonable range of $[0.1: 0.1: 10] \frac{\mathrm{mmol}}{\mathrm{g}}$ for the concentrations $c_{i}$ of all three components 
allows for $100^{3}$ different concentration vectors $\mathbf{c}=\left[c_{1}, c_{2}, c_{3}\right]^{T}$. Exploiting the general definition (A.3) of the Jacobian, every eigenvalue that corresponds to a particular vector $\mathbf{c}$ can be calculated numerically using standard software, e.g. MATLAB ${ }^{\circledR}[29]$. The results are shown in Fig. $7 \mathrm{~b}$, where the location of the eigenvalues $\lambda_{i}(\mathbf{c})$ in the complex right half-plane is shown. Since the existence of complex eigenvalues can be easily concluded from Fig. 17b, the Jacobian of this counter example is indeed not hyperbolic. Thus, also ruling out the stronger properties of the Jacobian to be strictly hyperbolic or having exclusively real, positive eigenvalues. 


\section{List of Tables}

1 Parameters of the first example. . . . . . . . . . . 27

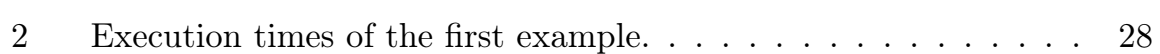

3 Parameters of the second example . . . . . . . . . . . . . 29

4 Parameters of the Tóth isotherm. . . . . . . . . . . . 30 


\begin{tabular}{|c|c|c|}
\hline parameter & value & description \\
\hline$L_{c}[\mathrm{~cm}]$ & 5 & column length \\
\hline$N_{z}[-]$ & 100 & number of grid points \\
\hline$u\left[\frac{\mathrm{cm}}{\mathrm{min}}\right]$ & 7.9556 & interstitial velocity \\
\hline$\epsilon[-]$ & 0.745 & void fraction \\
\hline$q_{11}^{\text {sat }}\left[\frac{\mathrm{mmol}}{\mathrm{l}}\right]$ & 147.2 & isotherm parameter \\
\hline$q_{12}^{\text {sat }}\left[\frac{\mathrm{mmol}}{\mathrm{l}}\right]$ & 6.0 & isotherm parameter \\
\hline$b_{11}\left[\frac{\mathrm{l}}{\mathrm{mmol}}\right]$ & 0.087 & isotherm parameter \\
\hline$b_{12}\left[\frac{\mathrm{l}^{2}}{\mathrm{mmol}}{ }^{2}\right]$ & 0.01 & isotherm parameter \\
\hline$b_{13}\left[\frac{\mathrm{l}}{\mathrm{mmol}}\right]$ & 1.69 & isotherm parameter \\
\hline$q_{21}^{\text {sat }}\left[\frac{\mathrm{mmol}}{\mathrm{l}}\right]$ & 122.4 & isotherm parameter \\
\hline$q_{22}^{\text {sat }}\left[\frac{\mathrm{mmol}}{\mathrm{l}}\right]$ & 17.0 & isotherm parameter \\
\hline$b_{21}\left[\frac{\mathrm{l}}{\mathrm{mmol}}\right]$ & 0.103 & isotherm parameter \\
\hline$b_{22}\left[\frac{\mathrm{l}^{2}}{\mathrm{mmol}}\right]$ & 0.032 & isotherm parameter \\
\hline$b_{23}\left[\frac{\mathrm{l}}{\mathrm{mmol}}\right]$ & 1.78 & isotherm parameter \\
\hline
\end{tabular}

Table 1: Parameters of the first example with explicit combined quadratic plus Langmuir SCIs. 


\begin{tabular}{|c|c|c|}
\hline formulation & solver & average execution time \\
\hline MOL-based DAE & IDAS & $5.2 \mathrm{~s}$ \\
& ODE15s & $11.0 \mathrm{~s}$ \\
\hline $\begin{array}{c}\text { full discretization } \\
\text { \&modified FastIAS }\end{array}$ & Newton-Raphson [13] & $12.0 \mathrm{~s}$ \\
\hline mixed DAE-FastIAS & ODE15s \& & $36.0 \mathrm{~s}$ \\
& Newton-Raphson [13] & \\
\hline Rothe-based DAE & ODE15s & $92.1 \mathrm{~s}$ \\
\hline
\end{tabular}

Table 2: Comparison of the execution times of the different numerical approaches for the first application example. 


\begin{tabular}{|c|c|c|}
\hline parameter & value & description \\
\hline$L_{c}[\mathrm{~m}]$ & 0.1 & column length \\
\hline$N_{z}[-]$ & 100 & number of grid points \\
\hline$u\left[\frac{\mathrm{m}}{\mathrm{s}}\right]$ & 0.0012 & interstitial velocity \\
\hline$\epsilon[-]$ & 0.8 & void fraction \\
\hline$q_{1}^{\text {sat }}\left[\frac{\mathrm{mg}}{\mathrm{ml}}\right]$ & 75.0 & isotherm parameter \\
\hline$q_{2}^{\text {sat }}\left[\frac{\mathrm{mg}}{\mathrm{ml}}\right]$ & 316.0 & isotherm parameter \\
\hline$b_{1}\left[\frac{\mathrm{ml}}{\mathrm{mg}}\right]$ & 0.03047 & isotherm parameter \\
\hline$b_{2}\left[\frac{\mathrm{ml}}{\mathrm{mg}}\right]$ & 0.0153 & isotherm parameter \\
\hline$\chi_{1}[-]$ & 0.6434 & isotherm parameter \\
\hline$\chi_{2}[-]$ & -2.037 & isotherm parameter \\
\hline
\end{tabular}

Table 3: Parameters of the second example with implicit Fowler-Guggenheim SCIs. 


\begin{tabular}{|c|ccc|}
\hline parameter & $i=1$ & $i=2$ & $i=3$ \\
\hline$q_{i}^{\text {sat }}\left[\frac{\text { mmol }}{g}\right]$ & 82.135 & 51.683 & 307.89 \\
\hline$b_{i}[-]$ & 22.476 & 6.894 & 307.89 \\
\hline$r_{i}[-]$ & 0.98 & 0.509 & 0.675 \\
\hline
\end{tabular}

Table 4: Parameters for the Tóth isotherm in the Appendix. 


\section{List of Figures}

1 SCIs of the first example. . . . . . . . . . . . . . . . . 32

2 Breakthrough curves for the first example . . . . . . . . . . . . . 33

3 Hodograph validation of the first example . . . . . . . . . . . . . 34

4 SCIs of the second example. . . . . . . . . . . . . . . . . . 35

5 Eluted peaks for the second example. . . . . . . . . . . . . 36

6 Hodograph validation of the second example. . . . . . . . . . . 37

7 Counter-example. . . . . . . . . . . . . . . . . . 38 


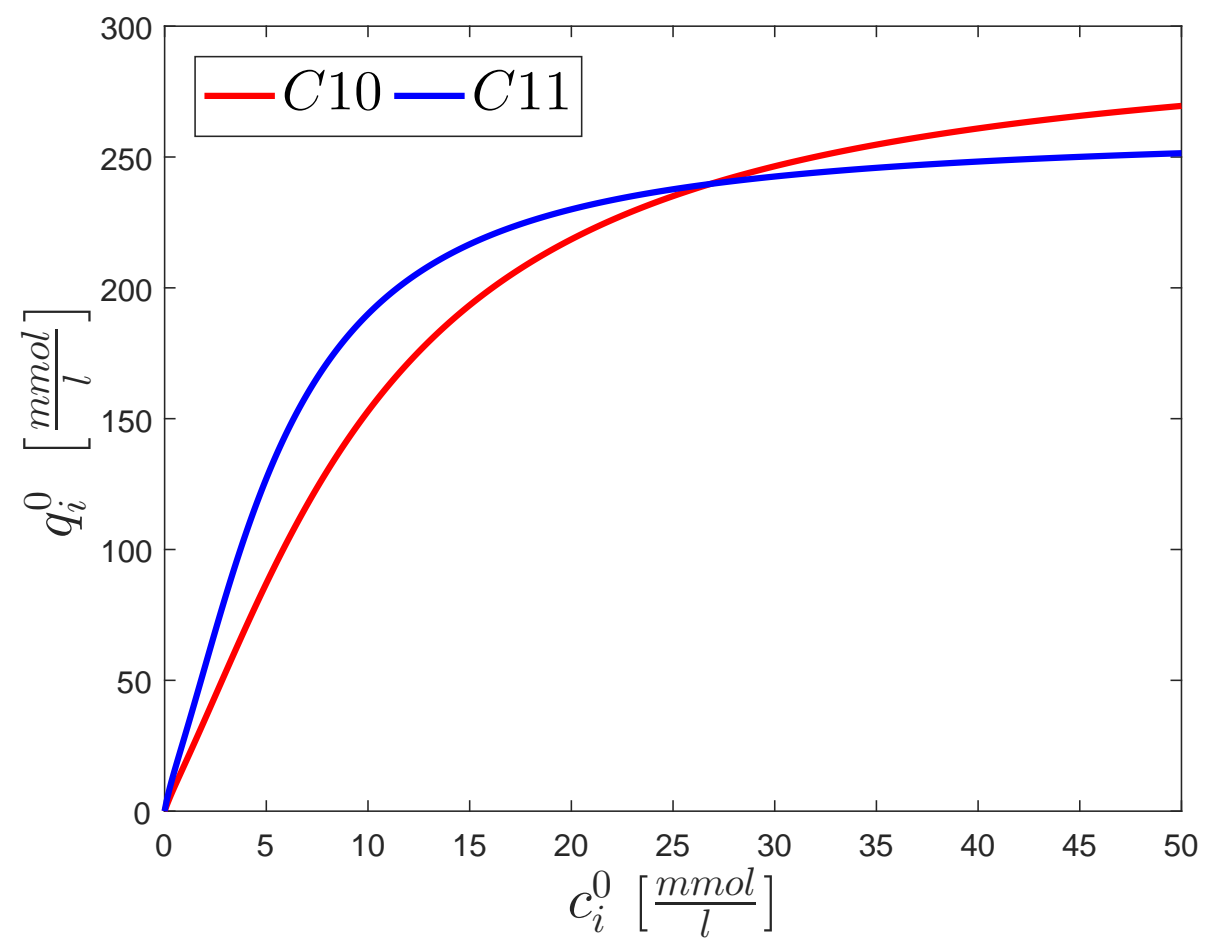

Figure 1: SCIs of phenyl-n-decane (C10) and phenyl-n-undecane (C11) computed with the combined quadratic plus Langmuir model. 


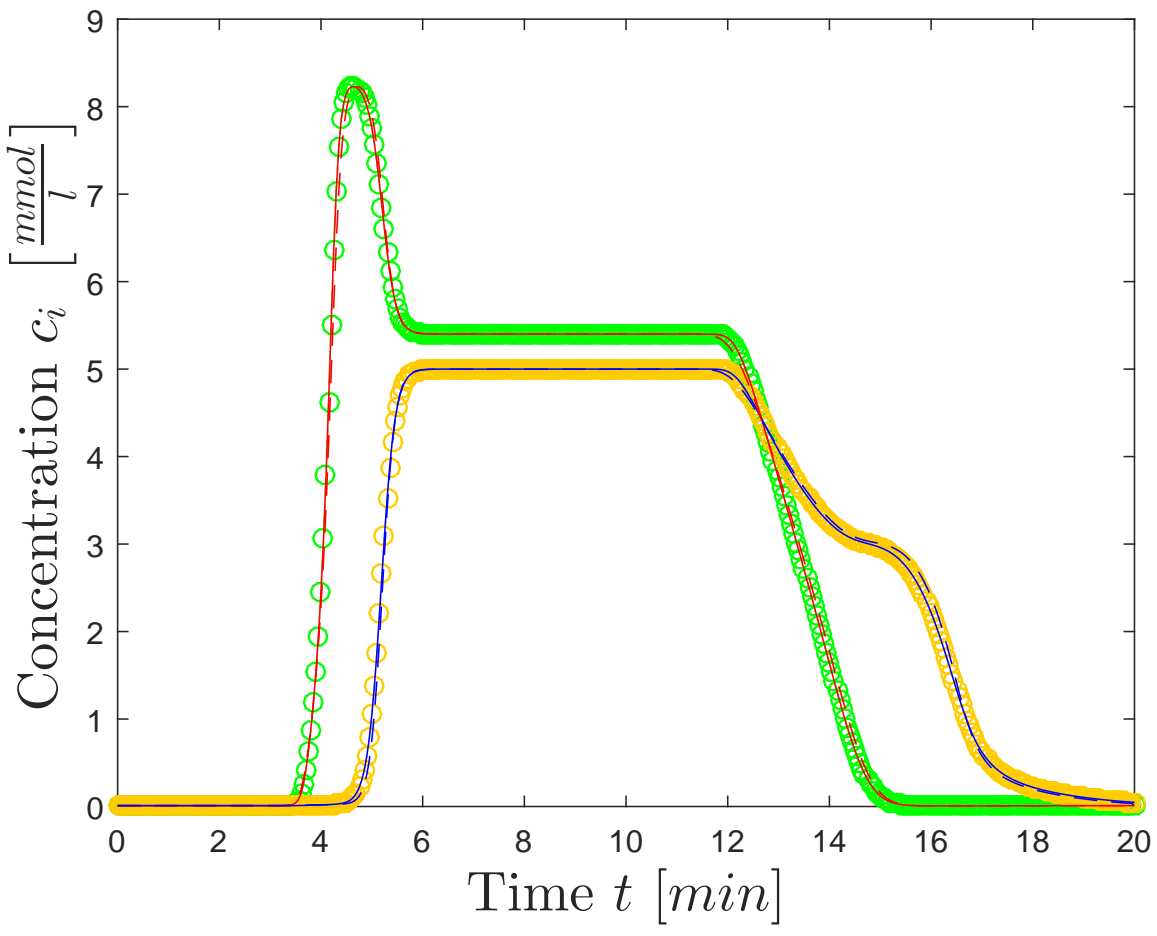

Figure 2: Calculated breakthrough curves for the first example. Solid lines - MOL-based (ODE15s and IDAS) and Rothe-Based DAE approach (ODE15s), circles - mixed DAE-FastIAS approach(Ode15s with Newton), dashed lines - modified FastIAS with total discretization (Newton). 
(a)

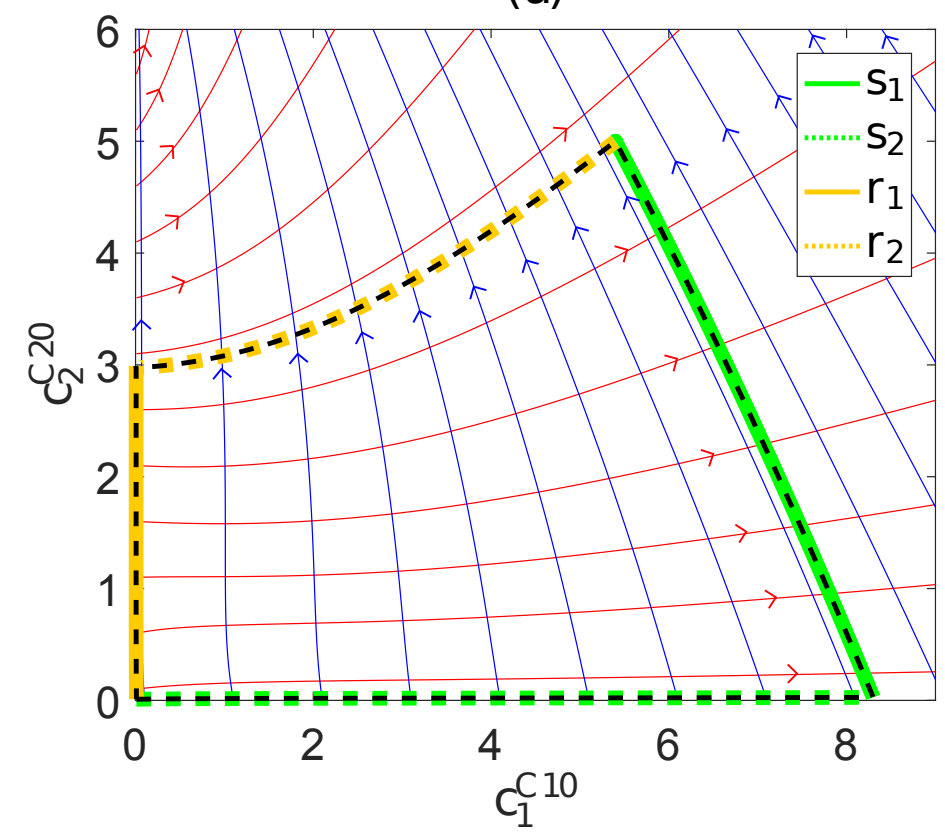

(b)

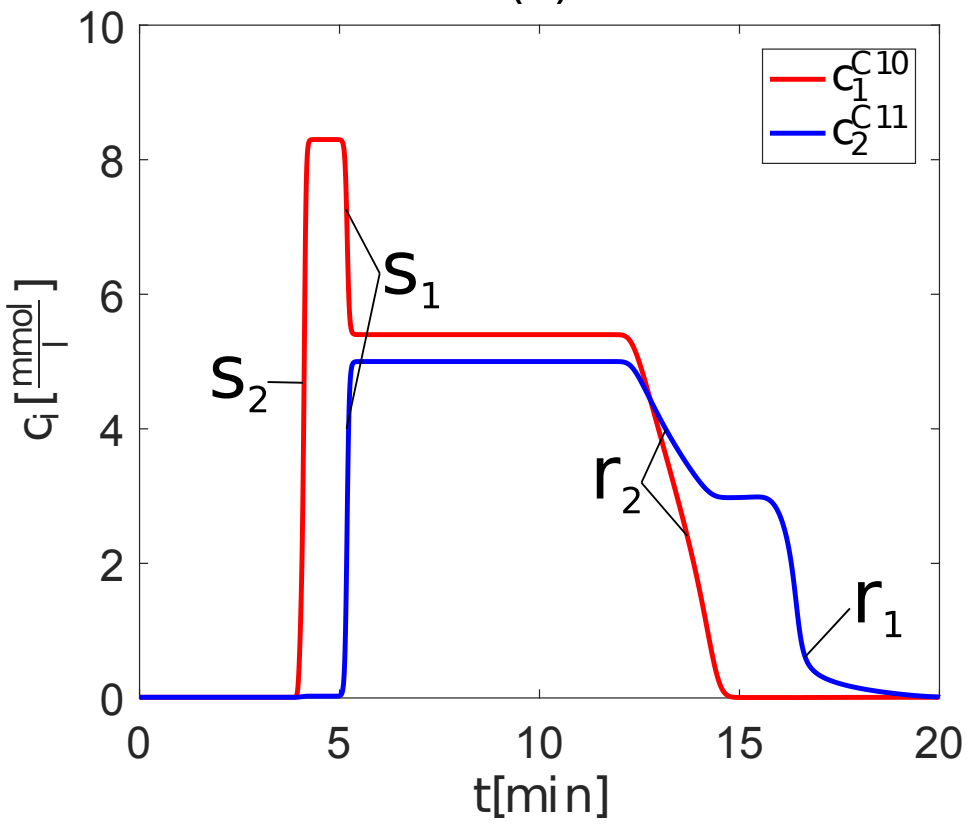

34

Figure 3: Validation of numerical results for the first example: (a) Trajectories in the hodograph space calculated with equilibrium theory (bold lines) overlaid with the numerical solution (dashed line). (b) Elution profiles of the numerical solution for $N_{z}=1000$ grid points. 


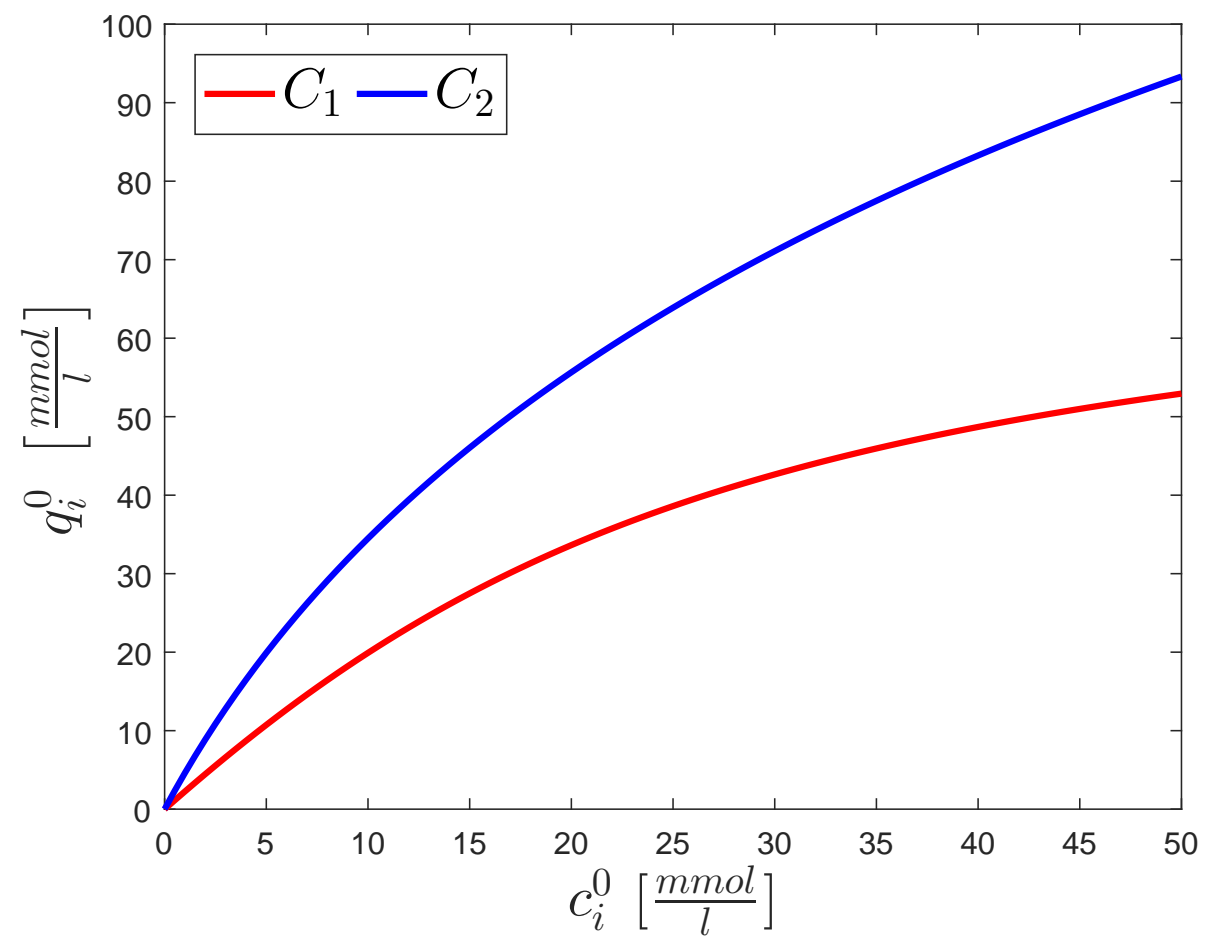

Figure 4: SCIs of 2-phenylethanol $\left(C_{1}\right)$ and 3-phenylpropanol $\left(C_{2}\right)$ computed with the FowlerGuggenheim model. 


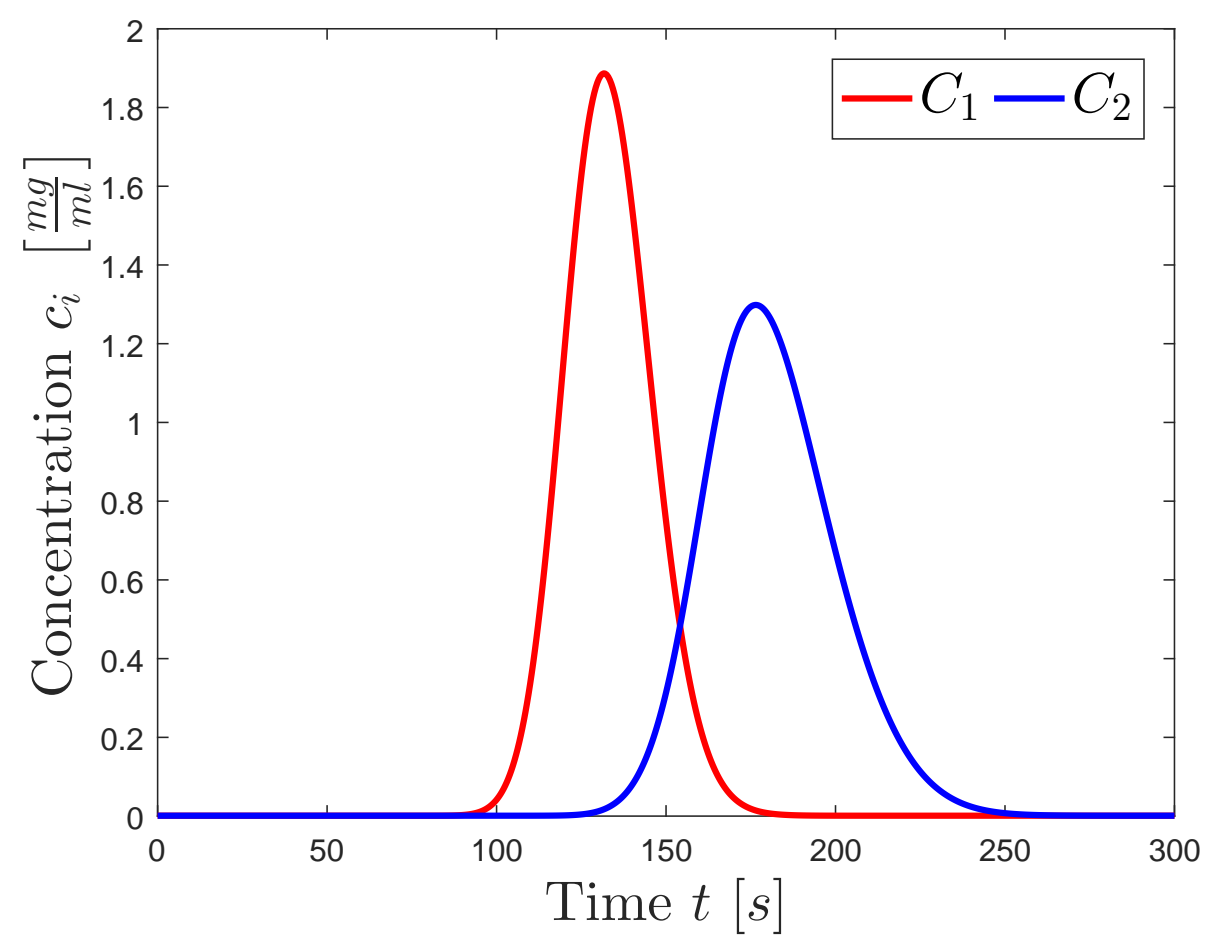

Figure 5: Calculated eluted peaks for the second example. 
(a)

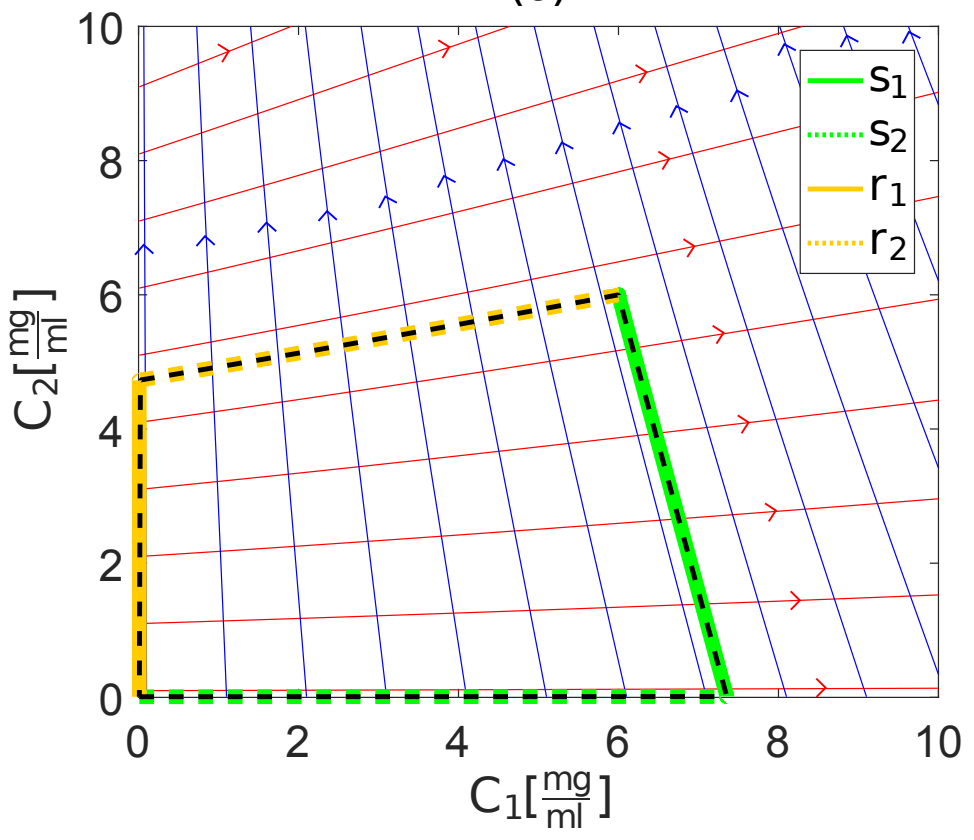

(b)

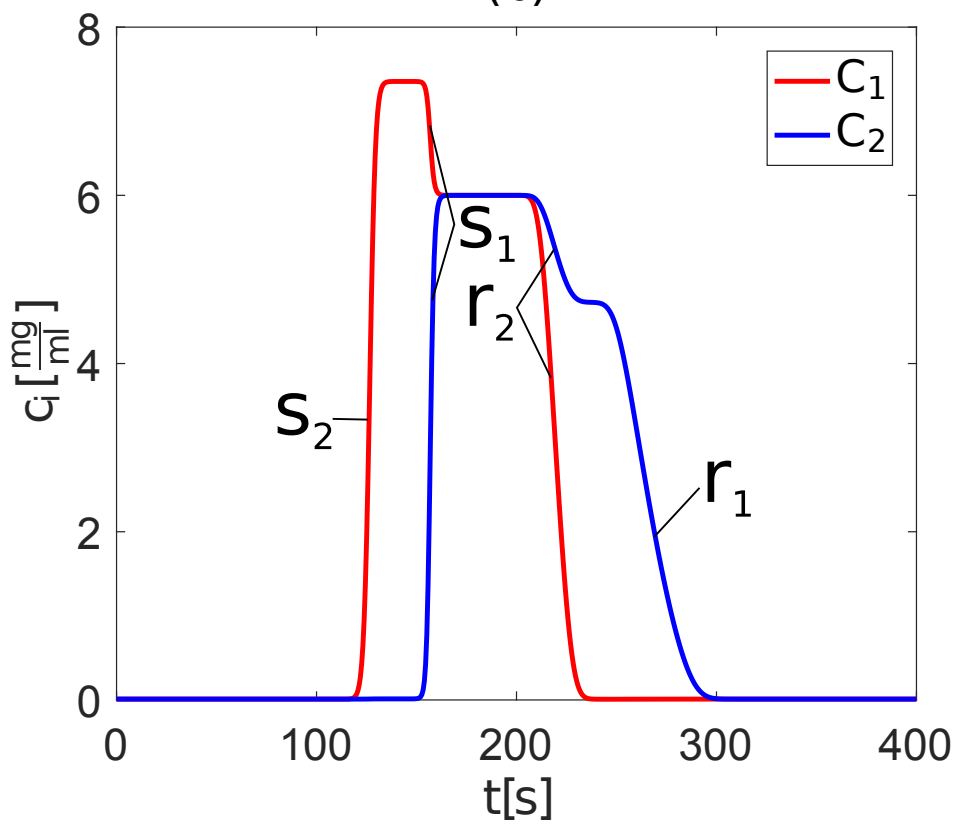

37

Figure 6: Validation of numerical results for the second example: (a) Trajectories in the hodograph space calculated with equilibrium theory (bold lines) overlaid with the numerical solution (dashed line). (b) Elution profiles of the numerical solution for $N_{z}=1000$ grid points and an injection interval of $[0,100] s$. 
(a)

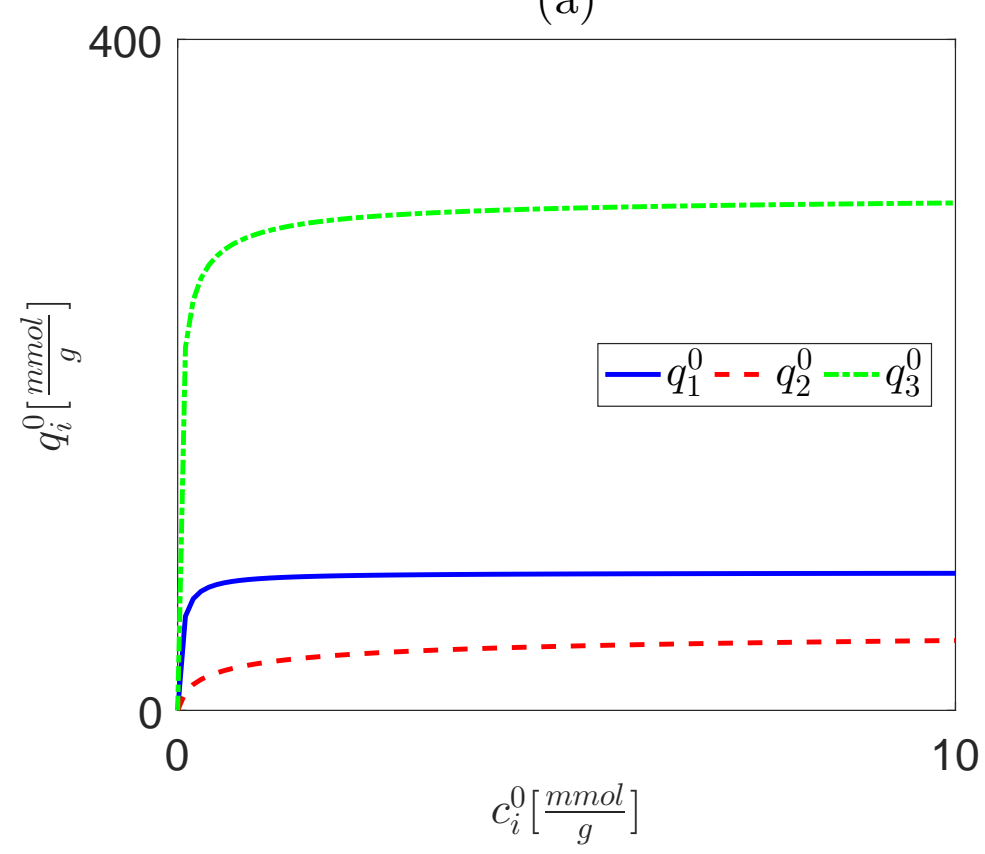

(b)

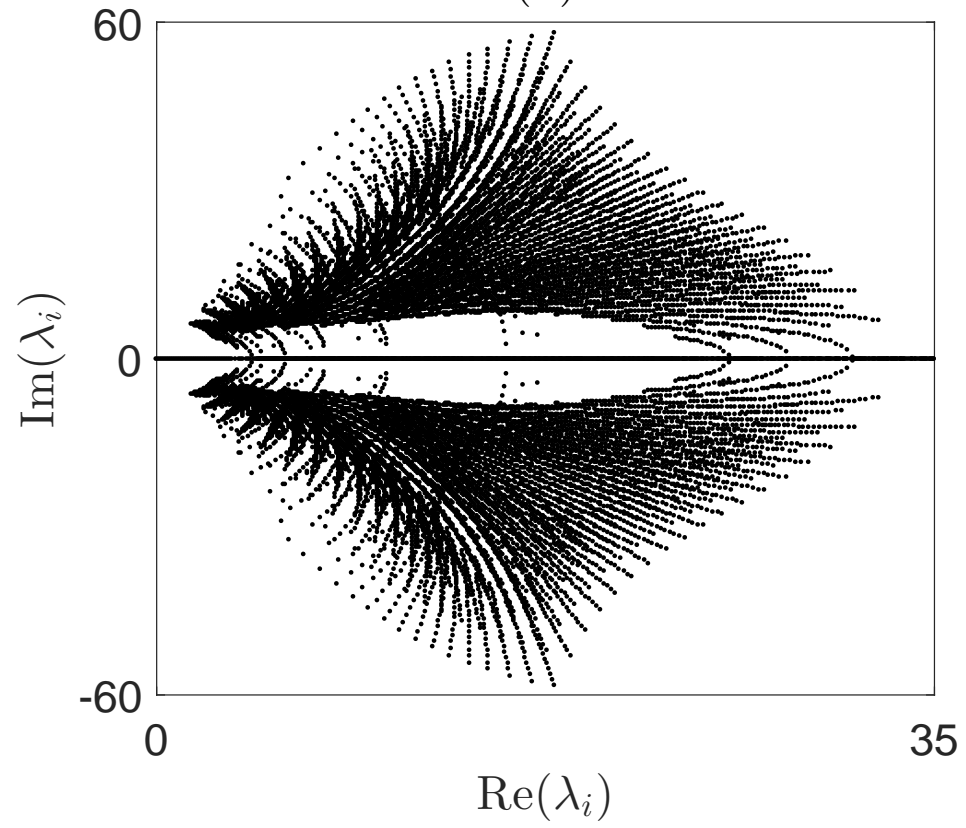

38

Figure 7: (a) SCIs of toluene (component '1'), dichloromethane (component '2') and trichloroethylene (component ' 3 ') computed with the Tóth model. (b) Complex right halfplane of all three eigenvalues for $100^{3}$ concentration combinations $c_{i} \in[0,10] \frac{\mathrm{mmol}}{\mathrm{g}}$. 